\title{
Statin medication in patients with epiretinal membrane is associated with low intravitreal EPO,TGF-beta-I, and VEGF levels
}

This article was published in the following Dove Press journal:

Clinical Ophthalmology

23 May 2016

Number of times this article has been viewed

\author{
Raimo Tuuminen' \\ Sirpa Loukovaara ${ }^{2}$ \\ 'Department of Ophthalmology, \\ Kymenlaakso Central Hospital, Kotka, \\ Finland; ${ }^{2}$ Unit of Vitreoretinal Surgery, \\ Department of Ophthalmology, \\ Helsinki University Hospital, \\ University of Helsinki, Helsinki, \\ Finland
}

Correspondence: Sirpa Loukovaara Department of Ophthalmology, Unit of Vitreoretinal Surgery, Helsinki University Hospital, Haartmaninkatu 4 C, FI-00290 Helsinki, Finland Email sirpa.loukovaara@hus.fi
Background: In eyes with idiopathic epiretinal membrane (iERM), the intravitreal growth factor and cytokine levels may associate with postvitrectomy outcomes. Here, we have analyzed the perioperative intravitreal protein levels of potent vasoactive, proinflammatory, and extracellular matrix-remodeling factors in iERM eyes and evaluated the postvitrectomy outcomes.

Methods: This was an institutional, observational study. Eyes operated on for iERM ( $\mathrm{n}=26)$ were analyzed according to the use of statin medication. Vitreous samples were subjected to protein measurements of angiopoietin- 1 and -2 , erythropoietin, transforming growth factor- $\beta 1$, and vascular endothelial growth factor by enzyme-linked immunosorbent assay, and of matrix metalloproteinase- 2 and -9 by gelatin zymography. One-month visual outcomes and 1-year revitrectomy rates were recorded.

Results: In iERM eyes of patients taking statins, intravitreal levels of erythropoietin (mean \pm standard deviation, $10.8 \pm 4.9$ vs $82.9 \pm 119.5 \mathrm{mIU} / \mathrm{mg}, P=0.003$ ), transforming growth factor- $\beta 1$ ( $2.3 \pm 4.7$ vs $15.8 \pm 16.3 \mathrm{pg} / \mathrm{mg}, P=0.035)$, and vascular endothelial growth factor $(5.5 \pm 9.9$ vs $236.6 \pm 491.6 \mathrm{pg} / \mathrm{mg}, P=0.006)$ were lower than in nonstatin-treated patients. At 1-month, visual gain did not significantly differ between iERM eyes of patients with statins and those without (improvement $0.27 \pm 0.20$ vs $0.16 \pm 0.38$ logarithm of the minimum angle of resolution units, $P=0.118$ ).

Conclusion: Systemic statin therapy might have a favorable effect on intravitreal factors involved in vascular permeability, inflammation, and fibroproliferation in aging human iERM eyes.

Keywords: epiretinal membrane, erythropoietin, HMG-CoA reductase inhibitors, transforming growth factor-beta, vascular endothelial growth factor, vitrectomy

\section{Introduction}

Despite advances in vitreoretinal microsurgery techniques over the years, epiretinal gliosis, such as idiopathic epiretinal membrane (iERM, also referred to as macular pucker), is a potentially sight-threatening vitreoretinal disorder, with aging being the principal nonmodifiable risk factor. ${ }^{1,2}$ Epidemiological studies have reported a prevalence of $1.02 \%-28.9 \%$ among different ethnic groups for iERM. ${ }^{3,4}$ Epiretinal gliosis on the macular surface ultimately leads to the formation of contractile tractional fibrocellular membranes, the development of severe retinal surface wrinkling, macular vascular distortion, and the breakdown of the blood-retinal barrier at the retinal pigment epithelial level. Limited understanding of the pathophysiology behind this vision-threatening vitreoretinal interface disease is hindering the development of nonsurgical treatment modalities. 
iERM involves inflammation, immune response, for example, classical and alternative pathway of complement activation, proteolysis, and cytoskeleton remodeling. ${ }^{5-7}$ Histologically, epiretinal membrane (ERM) is composed of multiple retinal and extraretinal cell types and extracellular matrix (ECM) proteins. Most internal limiting membrane (ILM) specimens removed from iERMs contain activated cells of glial origin (Müller cells, astrocytes, and microglia), hyalocytes, macrophages, fibroblasts, myofibroblasts, and retinal pigment epithelial cells, as well as ECM components, including newly formed collagens. ${ }^{8,9}$ Activated astro- and microglial cells have been also implicated as the key cell types involved in central nervous system (CNS) scar formation, and neuroinflammation and degeneration in the human retina. ${ }^{10-12}$

Fibrosis in the CNS and the vitreoretinal interface, an abnormal wound-healing process, is often related to ischemia (eg, vasoconstriction predisposed by smoking) and neuroinflammation. This involves upregulation of proinflammatory and profibrotic cytokines, adhesion molecules, and growth factors. Matrix metalloproteinases (MMPs), a group of zinc- and calcium-dependent proteinases, degrade components of the ECM, with MMP-2 and -9 being especially associated with the development of postoperative proliferative vitreoretinopathy (PVR). ${ }^{13}$ Transforming growth factor (TGF)- $\beta$, a multifunctional cytokine, is implicated in both barrier breakdown-induced permeability and fibroproliferation-induced contraction of PVR membranes. ${ }^{14,15}$ Moreover, vascular endothelial growth factor (VEGF) is linked to both vascular and avascular PVR membranes, and anti-VEGF therapies have shown a prophylactic effect against postoperative PVR formation. ${ }^{16,17}$ Angiopoietins (ANGPTs), potent regulators of endothelial cell-cell and cell-ECM interactions, were recently suggested as potential therapeutic targets in both neurotrauma and ischemia-reperfusion studies. ${ }^{18,19}$

Statins, 3-hydroxy-3-methyl-glutaryl-coenzyme A reductase inhibitors, were shown to reduce neuroinflammation and astrocyte activation in CNS injuries and enhance ganglion cell survival of ischemic rat retinas. ${ }^{20-22}$ In eyes subjected to surgery for fibroproliferative vitreoretinal disease, microvascular- and neuroprotective actions of statins ${ }^{23-25}$ might modulate integrity of the blood-retinal barrier, retinal wound healing, inflammation-related PVR, and photoreceptor survival. ${ }^{26,27}$

In the present study, we investigated the intravitreal levels of vasoactive, proinflammatory, and fibrotic factors; the visual outcomes; and the probability of reoperations in a group of vitrectomized iERM patients on statin medication compared with patients without preoperative statin medication.

\section{Materials and methods Study design}

Patients were admitted for primary vitrectomy for management of iERM in the Unit of Vitreoretinal Surgery, Helsinki University Central Hospital, Helsinki, Finland. The study was conducted according to the tenets of the Declaration of Helsinki and was approved by the Institutional Review Board of Helsinki University Central Hospital. Signed informed consent was obtained from each participant before sampling. Confidentiality of the patient records was maintained when the clinical data were entered into a computer-based standardized data entry for analysis.

\section{Patients and controls}

In this study, a total of 26 eyes of 25 patients who underwent primary vitrectomy for iERM between 2006 and 2008 were studied. Diagnosis of iERM was performed using optical coherence tomography. Data were further analyzed according to the use or nonuse of lipid-lowering statin therapy. Of the 26 cases, 12 were on statin treatment (nine on lipophilic simvastatin and three on lipophilic atorvastatin), and 14 were without any statins. Exclusion criteria were other retinal comorbidities such as diabetic retinopathy, inflammatory or retinal vascular disorder, or trauma. The clinical characteristics and concomitant interfering medication of the study patients are presented in Tables 1 and 2. Time of exposure to statin therapy was not documented in our study. The decision for statin therapy was taken by the general practitioner or internal medicine doctor.

\section{Surgery}

Undiluted vitreous samples (up to $1,000 \mu \mathrm{L}$ ) were collected at the start of the standard three-port pars plana vitrectomy (20 or 23 Gauge, Accurus, Alcon Instruments, Inc, Fort Worth, TX, USA) without an infusion of artificial fluid. The samples were collected by manual aspiration into a syringe via the vitrectomy with the cutting function activated. Samples were transferred into sterile $1.5 \mathrm{~mL}$ Eppendorf tubes and immediately frozen at $-70^{\circ} \mathrm{C}$ until laboratory analysis.

The surgical technique consisted of a standard threeport pars plana vitrectomy. If the vitreous was attached to the posterior retina, posterior vitreous detachment was induced by suction with the vitrectomy probe over the optic disc. To visualize and identify the posterior hyaloid, ERMs and the ILM, intravitreal vital dyes were used (chromovitrectomy). Diluted indocyanine green and/or 
Table I Baseline characteristics

\begin{tabular}{|c|c|c|c|}
\hline Patient and ocular variables & Nonstatin $(n=14)$ & Statin $(n=12)$ & $P$-value \\
\hline Age (years) & $69.5 \pm 7.0$ & $69.9 \pm 4.6$ & 0.667 \\
\hline Male:female, n (\%) & $7: 7(50: 50)$ & $3: 9(25: 75)$ & 0.191 \\
\hline BMI $\left(\mathrm{kg} / \mathrm{m}^{2}\right)$ & $29.9 \pm 12.0$ & $27.5 \pm 3.9$ & 0.979 \\
\hline ASA class $(1-4)$ & $2.5 \pm 0.5$ & $2.7 \pm 0.5$ & 0.084 \\
\hline Smoking, n (\%) & $9(64)$ & $7(58)$ & 0.756 \\
\hline Systolic BP (mmHg) & $159.2 \pm 28.9$ & $149.2 \pm 21.8$ & 0.437 \\
\hline Diastolic BP (mmHg) & $83.1 \pm 11.5$ & $84.5 \pm 11.4$ & 0.574 \\
\hline Intraocular pressure $(\mathrm{mmHg})$ & $15.0 \pm 2.9$ & $14.5 \pm 3.4$ & 0.769 \\
\hline Pseudophakic (IOL), n (\%) & $4(29)$ & $6(50)$ & 0.263 \\
\hline
\end{tabular}

Notes: Data presented as mean \pm SD or absolute number and proportion. For two-group comparisons, qualitative data were analyzed by two-factor $\chi^{2}$ test and continuous variables by nonparametric Mann-Whitney U-test.

Abbreviations: ASA, American Society of Anesthesiologists; BMI, body mass index; BP, blood pressure; IOL, intraocular lens; SD, standard deviation.

Table 2 Concomitant medication of the study patients

\begin{tabular}{|c|c|c|c|}
\hline Medication & Nonstatin $(n=14)$ & Statin $(n=12)$ & $P$-value \\
\hline \multicolumn{4}{|l|}{ Hypercholesterolemia } \\
\hline Fibrate & I & 0 & 0.345 \\
\hline Statin (simvastatin:atorvastatin) & 0 & $12(9: 3)$ & \\
\hline \multicolumn{4}{|l|}{ Diabetes } \\
\hline Oral only:insulin only:combination & $3(1: 0: 2)$ & $2(2: 0: 0)$ & 0.759 \\
\hline \multicolumn{4}{|l|}{ Hypertension } \\
\hline ACE:AT2 & 3 & 4 & 0.495 \\
\hline$\beta$-Blocker & 5 & 6 & 0.462 \\
\hline Calcium channel blocker & 3 & 2 & 0.759 \\
\hline \multicolumn{4}{|l|}{ (vascular:cardioselective) } \\
\hline Diuretic & 2 & 3 & 0.490 \\
\hline \multicolumn{4}{|l|}{ Circulation } \\
\hline Anticoagulation & 0 & I & 0.271 \\
\hline Antithrombotic & 6 & 6 & 0.716 \\
\hline Inotropic & I & 0 & 0.345 \\
\hline Nitrate & 2 & 2 & 0.867 \\
\hline \multicolumn{4}{|l|}{ Central nervous system } \\
\hline Antidepressive & 0 & 3 & 0.047 \\
\hline Benzodiazepine & 0 & I & 0.271 \\
\hline Dementia & I & 0 & 0.345 \\
\hline Nonbenzodiazepine hypnotic & 0 & I & 0.271 \\
\hline \multicolumn{4}{|l|}{ Gastrointestinal tract } \\
\hline Proton pump inhibitor & 2 & 2 & 0.867 \\
\hline \multicolumn{4}{|l|}{ Other hormones and vitamins } \\
\hline $\mathrm{BPH}$ & I & I & 0.910 \\
\hline Hormonal substitution & 3 & 2 & 0.759 \\
\hline Thyroxin & I & 2 & 0.449 \\
\hline Vitamins and micronutrients* & 2 & 3 & 0.490 \\
\hline \multicolumn{4}{|l|}{ Immunomodulation } \\
\hline Antihistamines & 3 & I & 0.356 \\
\hline Glucocorticoid & 1 & 0 & 0.345 \\
\hline Glucocorticoid inhalated & 2 & 5 & 0.117 \\
\hline NSAIDs & 0 & I & 0.271 \\
\hline \multicolumn{4}{|l|}{ Infection } \\
\hline Antibiotic & I & 0 & 0.345 \\
\hline \multicolumn{4}{|l|}{ Topical medication } \\
\hline Allergy & I & 0 & 0.345 \\
\hline Glaucoma & 0 & I & 0.271 \\
\hline
\end{tabular}

Notes: Data presented as $\mathrm{N}$-value of concomitant preoperative medication of 14 eyes of 14 nonstatin and 12 eyes of eleven statin-treated patients. For two-group comparisons, qualitative data were analyzed by two-factor $\chi^{2}$ test. *Vitamins and micronutrients counted by prescription. $P \leq 0.05$ shows a statistically significant result between the groups.

Abbreviations: ACE, angiotensin converting enzyme inhibitor; AT2, angiotensin II receptor antagonist; BPH, benign prostatic hyperplasia; NSAIDs, nonsteroidal anti-inflammatory drugs. 
MembraneBlue-Dual ${ }^{\circledR}$ (D.O.R.C., Zuidland, the Netherlands) dye-assisted ERM \pm ILM peeling was performed. Air-fluid exchange and flushing of the vitreous cavity with $\mathrm{SF}_{6}$ (sulfur hexafluoride) was performed in six eyes (23.1\%), $\mathrm{C}_{2} \mathrm{~F}_{6}$ (hexafluoroethane) in ten eyes (38.5\%), and $\mathrm{C}_{3} \mathrm{~F}_{8}$ (octafluoropropane) in one eye (3.8\%). Nine (34.6\%) eyes were filled with balanced salt solution.

\section{Determination of biomarkers}

Frozen vitreous humor samples were thawed and clarified by centrifugation at $13,000 \mathrm{rpm}$ at room temperature for 5 minutes. A reference serum sample was used to calculate intra- and inter-assay variation. Standard curves were constructed by plotting the concentration and mean absorbance and the plates were analyzed using a Victor2 multilabel counter microplate reader (Wallac, Turku, Finland) at a wavelength of $450 \mathrm{~nm}$. To calculate the concentration, the standards were fitted with a second-order polynomial equation.

For ANGPT-1 and -2 measurements in vitreous samples, we used solid-phase enzyme-linked immunosorbent assay (ELISA) kits (DANG10 and DANG20, R\&D Systems, Minneapolis, MN, USA). Total TGF- $\beta 1$ and VEGF levels in the vitreous were measured using Quantikine ELISA kits (DB100B and DVE00, respectively, R\&D Systems). Latent forms of TGF- $\beta 1$ were acid-activated before the assay according to the manufacturer's instructions. Erythropoietin (EPO) levels in the vitreous were determined by a Quantikine human EPO IVD ELISA (DEP00, R\&D Systems).

Gelatin zymography was performed to evaluate total MMP-2 and -9 zymogens in the vitreous samples. To analyze the gelatinolytic proteins, aliquots of vitreous samples were subjected to gelatin zymography. ${ }^{28}$ Samples were dissolved in nonreducing Laemmli sample buffer and separated by sodium dodecyl sulfate polyacrylamide gel electrophoresis using $10 \%$ polyacrylamide gels containing $1 \mathrm{mg} / \mathrm{mL}$ of gelatin. After electrophoresis, the gels were washed twice with 50 $\mathrm{mM}$ Tris- $\mathrm{HCl}$, pH 7.6, containing $5 \mathrm{mM} \mathrm{CaCl}_{2}, 1 \mu \mathrm{M} \mathrm{ZnCl}_{2}$, $2.5 \%$ Triton X-100 (v/v) for 15 minutes to remove sodium dodecyl sulfate, followed by a brief rinse in washing buffer without Triton X-100. The gels were then incubated at $37^{\circ} \mathrm{C}$ for 12-24 hours in $50 \mathrm{mM}$ Tris- $\mathrm{HCl}$ buffer containing $5 \mathrm{mM}$ $\mathrm{CaCl}_{2}, 1 \mu \mathrm{M} \mathrm{ZnCl}_{2}, 1 \%$ Triton X-100, 0.02\% $\mathrm{NaN}_{3}, \mathrm{pH}$ 7.6. The gels were stained with Coomassie brilliant blue R250 followed by destaining with $10 \%$ acetic acid and 5\% methanol. The zymogen gels were later scanned by an image scanner, and the areas of clear bands corresponding to MMP-2 and -9 activity were calculated using ImageJ software (v1.42q, NIH, Bethesda, MD, USA) on nonaltered original Tagged Image
File Format files in the 8-bit dynamic range of signal intensities. The values are reported in arbitrary units $(\mathrm{AU} / \mathrm{mL})$.

\section{Total protein measurement}

Vitreous total protein concentrations $(\mathrm{mg} / \mathrm{mL})$ were measured to compare the undiluted vitreous catch between study groups using a bicinchonic acid protein assay kit (Pierce, Rockford, IL, USA) according to manufacturer's instructions. The intravitreal concentrations (ANGPT-1 and -2, VEGF, TGF- $\beta 1$ $[\mathrm{pg} / \mathrm{mL}]$; EPO [mIU/mL]; or MMP-2 and -9 [AU/mL]) in eyes with iERM are given in relation to total protein concentration $(\mathrm{mg} / \mathrm{mL})$ in the vitreous sample.

\section{Visual acuity testing}

Best-corrected visual acuity (BCVA) was measured in a single sitting under standardized low light conditions on a standard Snellen chart at an observation distance of $5 \mathrm{~m}$. When the largest optotype could not be correctly recognized, the classification was very low VA on a semiquantitative scale such as counting fingers, hand motion, light perception, or no light perception. For statistical purposes, the Snellen values were transformed to the equivalent logarithm of the minimum angle of resolution (LogMAR) units. Very low VA measurements were converted as follows: counting fingers 1.9 , hand motion 2.3, light perception 2.7, and no light perception 3.0 LogMAR. ${ }^{29}$

\section{Statistical analyses}

All data are presented as mean \pm standard deviation, except for the absolute number and proportion for the nominal scale, and data were analyzed with SPSS 15.0 (SPSS Inc, Chicago, IL, USA). For two-group comparisons, qualitative data were analyzed using the two-factor $\chi^{2}$ test and continuous variables with the nonparametric Mann-Whitney $U$-test. Associations between variables were analyzed by Pearson's correlation. The need of revitrectomy was analyzed using the log-rank test. Death and the end of follow-up at 1 year were used as censoring events. $P \leq 0.05$ was considered statistically significant.

\section{Results \\ Baseline characteristics of the patients and vitrectomized eyes}

The study groups were age- and sex-matched (Table 1). All the subjects were of Caucasian origin. Interestingly, the majority of patients $(58 \%-64 \%)$ were smokers in both study groups (Table 1). The concomitant medication of the patients was similar between the groups, except for the fact that statin-treated patients had a higher probability of concomitant antidepressive medication ( $P=0.047$, Table 2 ). 
Table 3 Intravitreal protein concentrations in nonstatin- and statin-treated iERM patients

\begin{tabular}{llll}
\hline & Nonstatin $(\mathbf{n}=\mathbf{|} \mathbf{4})$ & Statin $(\mathbf{n}=\mathbf{|} \mathbf{2})$ & $\boldsymbol{P}$-value \\
\hline Total protein & $3.3 \pm 2.3$ & $4.1 \pm 1.3$ & 0.095 \\
ANGPT-I & $27.8 \pm 39.1$ & $12.3 \pm 6.1$ & 0.145 \\
ANGPT-2 & $82.9 \pm 108.5$ & $31.8 \pm 22.0$ & 0.176 \\
EPO & $82.9 \pm 119.5$ & $10.8 \pm 4.9$ & 0.003 \\
MMP-2 & $1,710.0 \pm 1,185.7$ & $854.1 \pm 496.8$ & 0.072 \\
MMP-9 & $36.7 \pm 47.7$ & $43.8 \pm 77.5$ & 1.000 \\
TGF- $\beta$ I & $15.8 \pm 16.3$ & $2.3 \pm 4.7$ & 0.035 \\
VEGF & $236.6 \pm 491.6$ & $5.5 \pm 9.9$ & 0.006 \\
\hline
\end{tabular}

Notes: The intravitreal concentrations (ANGPT-I and -2, VEGF, TGF- $\beta$ I $[\mathrm{pg} / \mathrm{mL}]$ EPO $[\mathrm{mlU} / \mathrm{mL}]$; or MMP-2 and $-9[\mathrm{AU} / \mathrm{mL}]$ ) in eyes with iERM are given in relation to total protein concentration $(\mathrm{mg} / \mathrm{mL})$ in the vitreous sample. Data presented as mean \pm SEM. For two-group comparisons, a nonparametric Mann-Whitney U-test was used. $P \leq 0.05$ shows a statistically significant result between the groups.

Abbreviations: ANGPT, angiopoietin; EPO, erythropoietin; iERM, idiopathic epiretinal membrane; MMP, matrix metalloproteinase; SEM, standard error of mean TGF- $\beta$ I, transforming growth factor- $\beta$ I; VEGF, vascular endothelial growth factor.

\section{Intravitreal protein levels}

The intravitreal protein levels of EPO $(P=0.003$, Table 3$)$, TGF- $\beta 1$ ( $P=0.035$, Table 3$)$, and VEGF $(P=0.006$, Table 3$)$ were significantly lower in the statin-treated compared to nonstatin-treated patients with iERM. No difference emerged in the intravitreal ANGPT-1 and -2, or MMP-2 and -9 (Table 3), or the plasma ANGPT-1 and -2 (Table S1) protein levels between the study groups. Intravitreal EPO levels correlated significantly with those of intravitreal VEGF $(r=0.892, P<0.001)$ and TGF- $\beta 1(r=0.695, P=0.001)$ levels (data not shown).

\section{Postoperative outcomes}

Preoperatively, the mean BCVA of iERM eyes did not differ between statin- and nonstatin-treated study patients (Table 4). Both the 1-month mean postoperative BCVA $(P=0.053$, Table 4$)$ and BCVA gain $(P=0.118$, Table 4$)$ did not significantly differ between the statin- compared to nonstatin-treated patients.

Table 4 One-month mean BCVA and I2-month revitrectomy of the study eyes

\begin{tabular}{llll}
\hline & Nonstatin $(\mathbf{n}=\mid \mathbf{1 4})$ & Statin $(\mathbf{n}=\mathbf{|} 2)$ & $\boldsymbol{P}$-value \\
\hline BCVA (pre) & $0.76 \pm 0.43$ & $0.63 \pm 0.17$ & 0.527 \\
BCVA (I-month) & $0.61 \pm 0.32$ & $0.37 \pm 0.16$ & 0.053 \\
BCVA gain & $0.16 \pm 0.38$ & $0.27 \pm 0.20$ & 0.118 \\
Revitrectomy & 4 & 1 & 0.219 \\
(I2-month) & & &
\end{tabular}

Notes: Data presented as mean \pm SD or as number of eyes of observed events in the two groups. For two-group comparisons, continuous variables were analyzed by nonparametric Mann-Whitney U-test and hazard of revitrectomy was analyzed using log-rank test. BCVA are given as LogMAR units.

Abbreviations: BCVA, best-corrected visual acuity; LogMAR, logarithm of the minimum angle of resolution; SD, standard deviation.
The number of eyes requiring revitrectomy during a 12-month follow-up was one of 12 in the statin group (due to rhegmatogenous retinal detachment [RRD]) and four of 14 in the nonstatin group (one due to RRD, one due to inferior PVR and RRD, and two due to recurrent ERM formation) $(P=0.219$, Table 4$)$. All secondary RRD cases occurred within 3-5 weeks of postprimary vitrectomy; they were reoperated and the retina was successfully reattached with gas or silicone oil. No endophthalmitis or prolonged hypotony was noted in the study eyes during the 12-month follow-up period.

\section{Discussion}

The vitreoretinal interface disease $\mathrm{EERM}$ is a common age-associated sight-threatening disorder worldwide, with aging and the development of anomalous posterior vitreous detachment being the most important known risk factors. ${ }^{2}$ However, the underlying mechanisms of iERM formation remain unclear. Numerous studies have reported intravitreal biochemical factors associated with secondary puckers related to various posterior segment inflammatory diseases, venous occlusive diseases, diabetic retinopathy, RRD, retinopathy of prematurity, and vitreomacular traction syndromes in age-related macular degeneration with choroidal neovascularization or diabetic macular edema. However, reports of intravitreal biochemical factors in iERM formation are rather scarce. ${ }^{8}$ Thus, our finding that several intravitreal vasoactive, proinflammatory, and profibrotic biomarkers in iERM eyes seem to be regulated by statin could be of great clinical importance regarding future therapeutic approach.

The aging process in CNS is known to be associated with degenerative changes such as presence of oxidative, proteotoxic, and metabolic stresses. ${ }^{30}$ Glial cell responses seem to be similar in the brain and neural retina. ${ }^{12}$ Comorbidities, such as long-term smoking, systemic hypertension, hyperlipidemia, obesity, or ischemia with an inflammatory component, could also play a role in the development of these age-related eye diseases. Indeed, majority of our patients were current smokers. Lifetime exposure to smoking could be related to increased oxidative stress and free radical generation, both of which are known to serve as triggers for parainflammation, vascular dysfunction, and fibroproliferation at the CNS and retinal level. ${ }^{31,32}$ Depending on the genetic background, smoking may affect the reparative potential of eye fibroblasts and promote the activation of proinflammatory pathways. ${ }^{33}$ Modern brain imaging techniques, such as advanced magnetic resonance imaging or positron emission tomography, could be useful in correlating the results with fibrosis formation in iERM eyes to assess the extent of gliosis/ fibrosis formation in the CNS. 
Recent advances in proteomics, for example, have revealed that TGF- $\beta 1$ is involved in iERM formation. ${ }^{5}$ TGF- $\beta 1$ is known to be present at low levels in the adult CNS. Microglia, infiltrating monocytes/macrophages, platelets, and reactive astrocytes are the major sources of increased TGF- $\beta 1$ during the early degenerative, remodeling, and healing phases in CNS disorders, such as ischemia and trauma. ${ }^{34,35}$ In the CNS, TGF- $\beta 1$ can also act as a secondary mediator of injury and promote astrocyte activation together with interleukins, fibrinogen, nuclear factor kappa beta and signal transducer and activator of transcription 3 and stimulate them to transdifferentiate into contractile myofibroblasts. ${ }^{36,37}$ In our study, the fact that TGF- $\beta 1$ levels in the eyes of patients with statin therapy were decreased compared with the levels in the nonstatin group could be due to differences in tissue remodeling mechanisms, perhaps reduced wound healing activity and epithelial-to-mesenchymal transition in eyes operated for iERM..$^{14,38}$

At best, surgical intervention is the choice of treatment only for a proportion of iERM cases. Furthermore, both existing intraocular fibrosis may cause irreversible tissue injury per se in the sensitive neuroretina and surgical removal of ERM may damage the nerve fiber layer, preventing the recovery of visual acuity even after a successful surgical procedure. Pharmacological interventions targeting CNS fibrosis would be of great importance. Statin use may modulate expression of proteins and cytokines associated with wound healing and survival. ${ }^{39}$ Furthermore, the role of angiogenic and antiangiogenic factors in iERM (eg, soluble VEGF receptor [s-VEGFR-1]) remains a topic for further research. ${ }^{40}$ In the future, the use of proteomics and genetics might enable better understanding of the pathophysiology of these complex age-related vitreoretinal interface disorders and improve nonsurgical treatment modalities for them. ${ }^{41}$

The major strength of our study was its measurement of several intravitreal vasoactive, proinflammatory, and profibrotic biomarkers in patients admitted for primary vitrectomy for iERM. However, our study has several limitations. First, we did not excise the membranes for immunocytochemical or ultrastructural analysis, and the number of intravitreal specimens was limited in both groups. Second, our study design did not allow additional postoperative controls after 1 month, thus the follow-up does not provide the anatomical optical coherence tomography outcome results, and the documentation for need of reoperations is retrospective in nature. Our present preliminary findings, however, suggest that statin treatment might have a role in anti-inflammatory and antifibrotic process in the aging human eye.

\section{Conclusion}

To conclude, the pathogenesis of fibrosis formation in the $\mathrm{CNS}$ and the aging eye is poorly understood, but can represent the end result of inflammation, apoptosis, proliferation, and abnormal wound healing. Currently, treatment strategies are limited, consisting of ophthalmic follow-up or microsurgery with pars plana vitrectomy with membrane peeling. Understanding of the regulation of fibrosis and modification of the fibrotic process is crucial to improve the surgical outcome of eyes with iERM. With the aging of the global population and the increasing life expectancy, the need for prevention and/or novel therapeutic approaches for fibrotic vitreoretinal interface diseases is growing.

\section{Acknowledgments}

Publication of this article was supported by grants from the Finnish Eye Foundation, the Eye and Tissue Bank Foundation, the Mary and Georg C. Ehrnrooth Foundation, the Nissi Foundation, the Waldemar von Frenckell Foundation, the Friends of the Blind, and HUCH Clinical Research Grants (TKK4150 and TYH1325). The authors thank Mrs Anna Salvato and Mrs Paula Kaijanmäki for excellent technical assistance.

\section{Disclosure}

The authors report no conflicts of interest in this work.

\section{References}

1. Steel DH, Lotery AJ. Idiopathic vitreomacular traction and macular hole: a comprehensive review of pathophysiology, diagnosis, and treatment. Eye (Lond). 2013;27 (Suppl 1):S1-S21.

2. Bu SC, Kuijer R, Li XR, Hooymans JM, Los LI. Idiopathic epiretinal membrane. Retina. 2014;34(12):2317-2335.

3. Duan XR, Liang YB, Friedman DS, et al. Prevalence and associations of epiretinal membranes in a rural Chinese adult population: the Handan Eye Study. Invest Ophthalmol Vis Sci. 2009;50(5): 2018-2023.

4. Aung KZ, Makeyeva G, Adams MK, et al. The prevalence and risk factors of epiretinal membranes: the Melbourne Collaborative Cohort Study. Retina. 2013;33(5):1026-1034.

5. Pollreisz A, Funk M, Breitwieser FP, et al. Quantitative proteomics of aqueous and vitreous fluid from patients with idiopathic epiretinal membranes. Exp Eye Res. 2013;108:48-58.

6. Joshi M, Agrawal S, Christoforidis JB. Inflammatory mechanisms of idiopathic epiretinal membrane formation. Mediators Inflamm. 2013; 2013:192582.

7. Yu J, Feng L, Wu Y, et al. Vitreous proteomic analysis of idiopathic epiretinal membranes. Mol Biosyst. 2014;10(10):2558-2566.

8. Zhao F, Gandorfer A, Haritoglou C, et al. Epiretinal cell proliferation in macular pucker and vitreomacular traction syndrome: analysis of flatmounted internal limiting membrane specimens. Retina. 2013;33(1): 77-88.

9. Schumann RG, Eibl KH, Zhao F, et al. Immunocytochemical and ultrastructural evidence of glial cells and hyalocytes in internal limiting membrane specimens of idiopathic macular holes. Invest Ophthalmol Vis Sci. 2011;52(11):7822-7834. 
10. Sofroniew MV, Vinters HV. Astrocytes: biology and pathology. Acta Neuropathol. 2010;119(1):7-35.

11. Fernandez-Klett F, Priller J. The fibrotic scar in neurological disorders. Brain Pathol. 2014;24(4):404-413.

12. Bringmann A, Iandiev I, Pannicke $T$, et al. Cellular signaling and factors involved in Muller cell gliosis: neuroprotective and detrimental effects. Prog Retin Eye Res. 2009;28(6):423-451.

13. Kon CH, Occleston NL, Charteris D, Daniels J, Aylward GW, Khaw PT. A prospective study of matrix metalloproteinases in proliferative vitreoretinopathy. Invest Ophthalmol Vis Sci. 1998;39(8):1524-1529.

14. Kawahara S, Hata Y, Kita T, et al. Potent inhibition of cicatricial contraction in proliferative vitreoretinal diseases by statins. Diabetes. 2008;57(10):2784-2793

15. Kita T, Hata Y, Arita R, et al. Role of TGF-beta in proliferative vitreoretinal diseases and ROCK as a therapeutic target. Proc Natl Acad Sci USA. 2008;105(45):17504-17509.

16. Tsanou E, Ioachim E, Stefaniotou M, et al. Immunohistochemical study of angiogenesis and proliferative activity in epiretinal membranes. Int J Clin Pract. 2005;59(10):1157-1161.

17. Pennock S, Kim D, Mukai S, et al. Ranibizumab is a potential prophylaxis for proliferative vitreoretinopathy, a nonangiogenic blinding disease. Am J Pathol. 2013;182(5):1659-1670.

18. Chittiboina P, Ganta V, Monceaux CP, Scott LK, Nanda A, Alexander JS. Angiopoietins as promising biomarkers and potential therapeutic targets in brain injury. Pathophysiology. 2013;20(1):15-21.

19. Syrjala SO, Tuuminen R, Nykanen AI, et al. Angiopoietin-2 inhibition prevents transplant ischemia-reperfusion injury and chronic rejection in rat cardiac allografts. Am J Transplant. 2014;14(5):1096-1108

20. Holmberg E, Zhang SX, Sarmiere PD, Kluge BR, White JT, Doolen S. Statins decrease chondroitin sulfate proteoglycan expression and acute astrocyte activation in central nervous system injury. Exp Neurol. 2008;214(1):78-86.

21. Schmeer C, Gamez A, Tausch S, Witte OW, Isenmann S. Statins modulate heat shock protein expression and enhance retinal ganglion cell survival after transient retinal ischemia/reperfusion in vivo. Invest Ophthalmol Vis Sci. 2008;49(11):4971-4981.

22. Beziaud T, Ru Chen X, El Shafey N, et al. Simvastatin in traumatic brain injury: effect on brain edema mechanisms. Crit Care Med. 2011; 39(10):2300-2307.

23. Tuuminen R, Syrjala S, Krebs R, et al. Donor simvastatin treatment abolishes rat cardiac allograft ischemia/reperfusion injury and chronic rejection through microvascular protection. Circulation. 2011;124(10): $1138-1150$.

24. Ko ML, Chen CF, Peng PH, Peng YH. Simvastatin upregulates Bcl-2 expression and protects retinal neurons from early ischemia/reperfusion injury in the rat retina. Exp Eye Res. 2011;93(5):580-585.

25. Marcus MW, Muskens RP, Ramdas WD, et al. Cholesterol-lowering drugs and incident open-angle glaucoma: a population-based cohort study. PLoS One. 2012;7(1):e29724.

26. Tuuminen R, Sahanne S, Loukovaara S. Low intravitreal angiopoietin-2 and VEGF levels in vitrectomized diabetic patients with simvastatin treatment. Acta Ophthalmol. 2014;92(7):675-681.
27. Tuuminen R, Haukka J, Loukovaara S. Statins in rhegmatogenous retinal detachment are associated with low intravitreal angiopoietin-2, VEGF and MMP-2 levels, and improved visual acuity gain in vitrectomized patients. Graefes Arch Clin Exp Ophthalmol. 2015;253(10): $1685-1693$.

28. Lohi J, Lehti K, Westermarck J, Kahari VM, Keski-Oja J. Regulation of membrane-type matrix metalloproteinase- 1 expression by growth factors and phorbol 12-myristate 13-acetate. Eur J Biochem. 1996;239(2): 239-247.

29. Schulze-Bonsel K, Feltgen N, Burau H, Hansen L, Bach M. Visual acuities "hand motion" and "counting fingers" can be quantified with the Freiburg visual acuity test. Invest Ophthalmol Vis Sci. 2006;47(3): 1236-1240.

30. Salminen A, Ojala J, Kaarniranta K, Haapasalo A, Hiltunen M, Soininen H. Astrocytes in the aging brain express characteristics of senescence-associated secretory phenotype. Eur J Neurosci. 2011;34(1): $3-11$.

31. Galor A, Lee DJ. Effects of smoking on ocular health. Curr Opin Ophthalmol. 2011;22(6):477-482.

32. Colombo G, Clerici M, Giustarini D, et al. Pathophysiology of tobacco smoke exposure: recent insights from comparative and redox proteomics. Mass Spectrom Rev. 2014;33(3):183-218.

33. Pouwels SD, Heijink IH, Brouwer U, et al. Genetic variation associates with susceptibility for cigarette smoke-induced neutrophilia in mice. Am J Physiol Lung Cell Mol Physiol. 2015;308(7):L693-L709.

34. Lehrmann E, Kiefer R, Christensen T, et al. Microglia and macrophages are major sources of locally produced transforming growth factor-beta 1 after transient middle cerebral artery occlusion in rats. Glia. 1998;24(4): $437-448$.

35. Mabuchi T, Kitagawa K, Ohtsuki T, et al. Contribution of microglia/ macrophages to expansion of infarction and response of oligodendrocytes after focal cerebral ischemia in rats. Stroke. 2000;31(7):1735-1743.

36. Minchiotti S, Stampachiacchiere B, Micera A, et al. Human idiopathic epiretinal membranes express NGF and NGF receptors. Retina. 2008; 28(4):628-637.

37. Kang W, Hebert JM. Signaling pathways in reactive astrocytes, a genetic perspective. Mol Neurobiol. 2011;43(3):147-154.

38. Chen Z, Shao Y, Li X. The roles of signaling pathways in epithelialto-mesenchymal transition of PVR. Mol Vis. 2015;21:706-710.

39. Suzuki-Banhesse VF, Azevedo FF, Araujo EP, et al. Effect of atorvastatin on wound healing in rats. Biol Res Nurs. 2015;17(2):159-168.

40. Matsunaga N, Chikaraishi Y, Izuta H, et al. Role of soluble vascular endothelial growth factor receptor-1 in the vitreous in proliferative diabetic retinopathy. Ophthalmology. 2008;115(11):1916-1922.

41. Franco C, Hess S. Recent proteomic advances in developmental, regeneration, and cancer governing signaling pathways. Proteomics. 2015; 15(5-6):1014-1025. 


\section{Supplementary material}

Table SI Plasma protein concentrations in nonstatin- and statin-treated iERM patients

\begin{tabular}{llll}
\hline & Nonstatin $(\mathbf{n}=\mathbf{| 1})$ & Statin $(\mathbf{n}=\mathbf{|} \mathbf{2})$ & $P$-value \\
\hline ANGPT-I & $3,112.5 \pm 2,018.1$ & $3,888.4 \pm 2,517.2$ & 0.456 \\
ANGPT-2 & $2,297.3 \pm 725.8$ & $2,356.1 \pm 971.2$ & 1.000 \\
\hline
\end{tabular}

Notes: The plasma concentrations (ANGPT-I and $-2[\mathrm{pg} / \mathrm{mL}]$ ) in patients with iERM. Data presented as mean \pm SD. For two group comparisons, a nonparametric MannWhitney U-test was used.

Abbreviations: ANGPT, angiopoietin; iERM, idiopathic epiretinal membrane; SD, standard deviation.

\section{Publish your work in this journal}

Clinical Ophthalmology is an international, peer-reviewed journal covering all subspecialties within ophthalmology. Key topics include: Optometry; Visual science; Pharmacology and drug therapy in eye diseases; Basic Sciences; Primary and Secondary eye care; Patient Safety and Quality of Care Improvements. This journal is indexed on
PubMed Central and CAS, and is the official journal of The Society of Clinical Ophthalmology (SCO). The manuscript management system is completely online and includes a very quick and fair peer-review system, which is all easy to use. Visit http://www.dovepress.com/ testimonials.php to read real quotes from published authors. 